\title{
Orthorexia Nervosa: moving forward in the field
}

\author{
Benjamin Missbach $^{1}$ (1) $\cdot$ Friederike Barthels ${ }^{2}$
}

Received: 23 January 2017 / Accepted: 25 January 2017 / Published online: 10 February 2017

(C) Springer International Publishing Switzerland 2017

Eating has become more complicated. There is a fine line between healthy eating and eating pathologically healthy. While some people can arrange their eating behavior quite well using simple heuristics that guide their way to healthy eating, others find this more difficult ending up being highly preoccupied with questions like: What could I possibly eat from this buffet? How can I avoid unwanted foods and what should I eat next without breaking my self-set rules? Since dietitians have first recognized this-somewhat offbeat - eating behavior, research on Orthorexia Nervosa has been popularized by media mentions rather than by in-depth scientific discussions. Still, Orthorexia Nervosa is not included in any Diagnostic Manuals (DSM-5, ICD10), even though an increasing amount of informal case reports from dietitians reveal how many individuals arrive at counseling sessions displaying orthorexic tendencies that cause severe psychological strain.

To overcome some of the conceptual and methodological problems, with this Topical Collection on Orthorexia Nervosa in Eating and Weight Disorders-Studies on Anorexia, Bulimia, and Obesity, we aim to gain several new insights. First, we want to increase awareness within the scientific community and stimulate further research in this field. To date, a total of 47 published articles can be found

This article is part of the topical collection on Orthorexia

Nervosa.

Benjamin Missbach

b.missbach@gmail.com

1 Department of Nutritional Sciences, University of Vienna, Althanstraße 14, 1090 Vienna, Austria

2 Department of Experimental Psychology, Heinrich-HeineUniversity Düsseldorf, Universitätsstaße 1, Düsseldorf, Germany in PubMed, with $70 \%$ published within the last 5 years. ${ }^{1}$ While we acknowledge this trend, we especially aim at high-quality scientific research in this Topical Collection. Second, we want to provide a decisive hub for scientific discussion based on data investigating different perspectives of Orthorexia Nervosa. We urge all contributing researchers to provide a wide range of research studies reporting relevant case studies, the development of novel and valid diagnostic tools and to provide meta-evidence via systematic reviews. Third and most importantly, we aim to bring together key researchers to move forward in the field. With this said, we will be able to join forces and hopefully improve our knowledge regarding Orthorexia Nervosa.

\section{Compliance with ethical standards}

Conflict of interest The authors declare that they have no conflict of interest.

Ethical approval This article does not contain any studies with human participants or animals performed by any of the authors.

\footnotetext{
1 PubMed Search: 01/17/2017.
} 